\title{
Voronoi Diagrams of Random Lines and Flats
}

\author{
R. A. Dwyer \\ Department of Computer Science, North Carolina State University, \\ Raleigh, NC 27695-8206, USA \\ dwyer@csc.ncsu.edu
}

\begin{abstract}
It is proved that, for any fixed $d \geq 3$ and $0 \leq k \leq d-1$, the expected combinatorial complexity of the Euclidean Voronoi diagram of $n$ random $k$-flats drawn independently from the uniform distribution on $k$-flats intersecting the unit ball in $\mathbb{R}^{d}$ is $\Theta\left(n^{d /(d-k)}\right)$ as $n \rightarrow \infty$. A by-product of the proof is a density transformation for integrating over sets of $d+1 k$-flats in $\mathbb{R}^{d}$.
\end{abstract}

\section{Introduction}

Analyzing and efficiently computing Voronoi diagrams of various ilks has been a common pastime of computational geometers ever since Shamos and Hoey's classic paper [7] addressing Voronoi diagrams of points in the plane appeared two decades ago. Subsequent researchers too numerous to name here have considered sets of line segments or circles, alternative metrics, higher dimensions, higher orders, objects in motion, and other variations found applicable to motion-planning, illumination, visibility, and other optimization problems.

Recently Chew et al. [2] have analyzed Voronoi diagrams of lines in $\mathbb{R}^{3}$ as a "first step towards planning a high-clearance translational motion for a convex polyhedral robot in three dimensions." Concentrating on "convex distance functions" defined by polytopes, they show an $O\left(n^{2} \alpha(n) \log n\right)$ bound on the worst-case combinatorial complexity of the diagram of $n$ lines. Although the Euclidean metric is a convex distance function, it is defined by the ball rather than a polytope. According to Chew et al., $O\left(n^{3+\varepsilon}\right)$ is the best bound known for the Euclidean Voronoi diagram. (Neither bound is known to be tight, although the polytope bound is tight within $\Theta(\log n)$.)

This work focuses on an asymptotic analysis of the average combinatorial complexity of the Voronoi diagram of random lines in fixed dimension $d \geq 3$. The lines are drawn independently from the uniform distribution on all lines intersecting the unit $d$-ball, as described in Section 2. The result is summarized in the following theorem: 
Theorem 1. For any fixed $d \geq 3$, the expected combinatorial complexity of the Euclidean Voronoi diagram of $n$ random lines drawn independently from the uniform distribution on lines intersecting the unit ball in $\mathbb{R}^{d}$ is $\Theta\left(n^{d /(d-1)}\right)$ as $n \rightarrow \infty$.

Although the particular distribution analyzed here may be unlikely in practice, it may well be more indicative of "typical" complexity than the worst-case bounds (which, at any rate, are not yet known to be tight). It is reasonable to conjecture that these bounds hold for any absolutely continuous distribution, i.e., any distribution having a density with respect to the conventional measure on the space of all lines. Therefore, this result should be useful to those who seek to construct such diagrams for practical applications.

In the next section we introduce our notation and formalize our notion of "uniform distribution." Section 3 derives a density transformation that allows us to express a set of $d+1$ lines in terms convenient for our analysis, a significant by-product of the research. In Section 4 this transformation is applied to complete the proof of Theorem 1. The main result is extended to sets of random $k$-dimensional flats in Section 5; the more general bound is $\Theta\left(n^{d /(d-k)}\right)$. (The case $k=0$ was addressed in an earlier work [3].) Some final remarks and open questions occupy Section 6.

\section{Definitions and Preliminaries}

Let $\mathcal{L}_{n}=\left\{L_{1}, L_{2}, \ldots, L_{n}\right\}$ be a set of lines in $\mathbb{R}^{d}$ for some fixed $d \geq 3$. If $\delta(\cdot, \cdot)$ denotes the distance between points in $\mathbb{R}^{d}$, we can define the distance between point $P$ and line $L$ by

$$
\delta(P, L)=\min _{P^{\prime} \in L} \delta\left(P, P^{\prime}\right)
$$

If $V: \mathbb{R}^{d} \rightarrow 2^{\mathcal{L}}$ satisfies

$$
V(P)=\left\{L \in \mathcal{L}_{n} \mid \delta(P, L) \leq \delta\left(P, L^{\prime}\right), \forall L^{\prime} \in \mathcal{L}_{n}\right\},
$$

then the preimages of the subsets of $\mathcal{L}$ are the cells of the Voronoi diagram of $\mathcal{L}_{n}$. A $k$-dimensional cell is called a $k$-cell; 0 -cells and 1-cells are called vertices and edges, respectively. In general, most subsets have empty preimages. In fact, if the lines of $\mathcal{L}_{n}$ are in general position, the preimage of any subset with more that $d+1$ elements is empty, and every $k$-cell is the preimage of a $(d+1-k)$-subset of $\mathcal{L}$. (The lines are in general position if no $d+2$ lines are tangent to a common $d$-ball, and no two lines are parallel. General position obtains with probability 1 under our model of random line.)

The combinatorial complexity of the Voronoi diagram is the number of nonempty cells it has. From the foregoing discussion, it should be clear that the combinatorial complexity is $O\left(n^{d+1}\right)$ for fixed $d$ as $n \rightarrow \infty$. In fact, it is known to be $O\left(n^{3+\varepsilon}\right)$ when $d=3$ and even this bound is not known to be tight [2]. The following lemma states that a bound on the number of vertices of the Voronoi diagram gives a bound on its overall combinatorial complexity.

Lemma 1. Let $V_{n}$ be the number of Voronoi vertices of $\mathcal{L}_{n}$. If the lines of $\mathcal{L}_{n}$ are in general position, then the total number of cells is at most $2^{d+1} V_{n}$. 
Proof. We use the analog of the "growing spheres" associated with Voronoi diagrams of point sites: We imagine cylinders growing around each line, each with radius $t$ at time $t$. If some $k<d+1$ lines define a Voronoi $(d-k+1)$-cell, then at some time $t^{\prime}$ there is a point $P$ lying on the surfaces of the $k$ lines' cylinders and outside every other line's cylinder. For all times $t \geq t^{\prime}$, these $k$ cylinders will have a nonempty intersection. As the other $n-k$ cylinders grow, each will eventually meet the intersection of the $k$. The first to do so joins the other $k$ lines to define a $(d-k)$-cell of the diagram. In this way the original set of $k$ lines can be repeatedly augmented to a set of $d+1$ lines forming a Voronoi vertex. Each such set has only $2^{d+1}$ subsets.

The usual Lebesgue measure on $\mathbb{R}^{k}$ ( $k$-volume) is denoted by $\lambda_{k}$. Slightly abusively, we use $B_{d}$ to denote both the unit $d$-ball and its volume, so that

$$
B_{d}=\lambda_{d}\left(B_{d}\right)=\frac{2 \pi^{d / 2}}{d \Gamma(d / 2)} .
$$

We use $\sigma_{k-1}$ to denote the usual rotation-invariant measure of orientations in $\mathbb{R}^{k}$. This may also be viewed as a measure on the space of (unit) vectors on the $(k-1)$-dimensional unit sphere (the boundary of the unit $k$-ball). $S_{d-1}$ denotes both the unit $(d-1)$-sphere and its $(d-1)$-volume ("surface area"), thus

$$
S_{d-1}=\int_{S_{d-1}} d \sigma_{d-1}(u)=d \cdot B_{d}=\frac{2 \pi^{d / 2}}{\Gamma(d / 2)} .
$$

Now we describe $\mu$, the uniform measure on the space of lines in $\mathbb{R}^{d}$. To choose a random line, we first choose its orientation uniformly, i.e., according to measure $\sigma_{d-1}$. A random line with fixed orientation is determined by its intersection with the unique perpendicular hyperplane passing through the origin. We choose an intersection point on the hyperplane according to $\lambda_{d-1}$, the uniform measure on $\mathbb{R}^{d-1}$. Thus if $P \in \mathbb{R}^{d-1}$ is the intersection and $u$ is a unit $d$-vector,

$$
d \mu(L)=d \sigma_{d-1}(u) d \lambda_{d-1}(P)
$$

is rotation- and translation-invariant and-up to a constant factor-uniquely so [6, p. 204]. To derive a uniform probability distribution, we restrict this measure to lines intersecting the unit $d$-ball. Then

$$
\int_{|P| \leq 1} \int_{|u|=1} d \sigma_{d-1}(u) d \lambda_{d-1}(P)=S_{d-1} B_{d-1}
$$

is bounded, and we have a uniform probability density of $\left(S_{d-1} B_{d-1}\right)^{-1}$.

Alternatively, we can express $P$ in spherical coordinates, i.e., in term of an orientation $v$ in $\mathbb{R}^{d}$ plus its distance $r$ from the origin. Since

$$
d \lambda_{d-1}(P)=r^{d-2} d r d \sigma_{d-2}(v),
$$

an equivalent method for drawing random lines intersecting the unit ball is to

(1) Select an orientation $u$ for the line segment joining the origin to the nearest point on the random line. 
(2) Select a distance $r$ from the origin to the line according to the (nonuniform) measure $r^{d-2}$.

(3) Select $v$, the orientation of the line on the hyperplane defined by $u$ and $r$, according to measure $\sigma_{d-2}$.

This view provides a more intuitive framework for some of our argumentation.

In order to analyze the expected number of Voronoi vertices, it is necessary to express the $d+1$ lines defining the vertex in terms of the vertex itself rather than in terms of the origin of the standard coordinate system. In the next section we describe such a system of coordinates for the lines and derive the corresponding density transformation. The density transformation could be derived by considering the $\left(2 d^{2}-2\right) \times\left(2 d^{2}-2\right)$ Jacobian determinant of the coordinate transformation, as was done elsewhere for $d+1$ points [3]. Instead, we use the more concise notations and techniques of the exterior algebra of differential forms, as presented by Santalo [6, Chapter 12], to carry out this task. This notation centers around the anticommutative wedge product, which satisfies

$$
d x \wedge d y=-d y \wedge d x \text { and (therefore) } d x \wedge d x=0 .
$$

To illustrate briefly, we rederive the well-known density transformation $d x d y=r d r d \theta$ relating Cartesian and polar coordinates in the plane. Since $x=r \cos \theta$ and $y=r \sin \theta$, we have

$$
\begin{aligned}
d x \wedge d y= & (-r \sin \theta d \theta+\cos \theta d r) \wedge(r \cos \theta d \theta+\sin \theta d r) \\
= & (-r \sin \theta d \theta) \wedge(r \cos \theta d \theta)+(-r \sin \theta d \theta) \wedge(\sin \theta d r) \\
& +(\cos \theta d r) \wedge(r \cos \theta d \theta)+(\cos \theta d r) \wedge(\sin \theta d r) \\
= & 0+\left(r \sin ^{2} \theta d r \wedge d \theta\right)+\left(r \cos ^{2} \theta d r \wedge d \theta\right)+0 \\
= & r d r \wedge d \theta .
\end{aligned}
$$

Using this technique and the fact that, for unit vector $u=\left(u^{(1)}, u^{(2)}, \ldots, u^{(d)}\right)$, we have

$$
d \sigma_{d-1}(u)=\frac{1}{u^{(1)}} \bigwedge_{i=2}^{d} d u^{(i)}
$$

it is not difficult to verify that

$$
d \sigma_{d-1}(u)=\sin ^{d-2} \psi d \psi d \sigma_{d-2}\left(u^{\prime}\right),
$$

where $\psi$ is the angle between $u$ and the positive $x^{(d)}$-axis, and $u^{\prime}$ is a unit vector in the hyperplane $x^{(d)}=0$.

\section{Density Transformations}

This section describes a convenient system of coordinates for expressing the $d+1$ lines defining a Voronoi vertex and the density transformation corresponding to these coordinates. 
Let $u_{i}$ be a unit vector parallel to line $L_{i}$, and let $H_{i}=\left\{x \mid\left\langle u_{i}, x\right\rangle=0\right\}$ be the hyperplane through the origin perpendicular to $L_{i}$. Let $\left(e_{i 1}, e_{i 2}, \ldots, e_{i, d-1}\right)$ be a moving frame, i.e., an orthonormal basis for $H_{i}$ in which each $e_{i j}$ is a continuous function of $u_{i}$. (For concreteness, we may use Raynaud's moving frame [5, p. 36].) Let $P_{i} \in \mathbb{R}^{d}$ be the vector of coordinates of $L_{i} \cap H_{i}$ with respect to the standard basis, and let $Q_{i} \in \mathbb{R}^{d-1}$ be its coordinates with respect to the moving frame, so that $Q_{i}^{(j)}=\left\langle P_{i}, e_{i j}\right\rangle$. Then, as discussed in Section 2,

$$
d \mu\left(L_{i}\right)=d \sigma_{d-1}\left(u_{i}\right) \wedge d \lambda_{d-1}\left(Q_{i}\right)=d \sigma_{d-1}\left(u_{i}\right) \wedge d Q_{i}^{(1)} \wedge d Q_{i}^{(2)} \wedge \cdots \wedge d Q_{i}^{(d-1)}
$$

Now each vertex of the Voronoi diagram of the lines is (with probability 1) equidistant from $(d+1)$ lines. If $C$ is the vertex and $r$ is the distance to each line, then the $d+1$ lines are tangent to a $(d-1)$-sphere of radius $r$ centered at $C$.

Let $\bar{H}_{i}=\left\{x \mid\left\langle u_{i}, x\right\rangle=\left\langle u_{i}, C\right\rangle\right\}$ be the hyperplane perpendicular to line $L_{i}$ passing through $C$. Let $\vec{P}_{i}$ be the vector of coordinates of $\left(L_{i} \cap \bar{H}_{i}\right)-C$ with respect to the standard basis for $\mathbb{R}^{d}$, and let $\bar{Q}_{i}$ be its coordinates with respect to the moving frame. Let $v_{i}=\bar{Q}_{i} /\left|\bar{Q}_{i}\right|$ be a unit vector in $\mathbb{R}^{d-1}$. Then

$$
Q_{i}^{(j)}=\left\langle C, e_{i j}\right\rangle+\bar{Q}_{i}^{(j)}=\left\langle C, e_{i j}\right\rangle+r v_{i}^{(j)}
$$

and

$$
d Q_{i}^{(j)}=\left\langle C, d e_{i j}\right\rangle+\left\langle d C, e_{i j}\right\rangle+r \cdot d v_{i}^{(j)}+v_{i}^{(j)} \cdot d r .
$$

Our goal is to use these equations to express

$$
\bigwedge_{i=0}^{d} d \mu\left(L_{i}\right)=\bigwedge_{i=0}^{d}\left(d \sigma_{d-1}\left(u_{i}\right) \wedge d Q_{i}^{(1)} \wedge d Q_{i}^{(2)} \wedge \cdots \wedge d Q_{i}^{(d-1)}\right)
$$

in terms of the $d u_{i}^{(j)}, d C^{(j)}, d r$, and $d v_{i}^{(j)}$.

Since our density is translation-invariant, we may assume without loss of generality that $C=(0,0, \ldots, 0)$ and write

$$
d Q_{i}^{(j)} \equiv\left\langle e_{i j}, d C\right\rangle+r \cdot d v_{i}^{(j)}+v_{i}^{(j)} \cdot d r
$$

where "I" means that the right side may be substituted for the left in our derivation.

Now we define

$$
\begin{aligned}
\Sigma_{i} & =\sum_{j=1}^{d-1} v_{i}^{(j)} d Q_{i}^{(j)} \\
& \equiv \sum_{j=1}^{d-1}\left\langle v_{i}^{(j)} e_{i j}, d C\right\rangle+r \sum_{j=1}^{d-1} v_{i}^{(j)} d v_{i}^{(j)}+\sum_{j=1}^{d-1}\left(v_{i}^{(j)}\right)^{2} \cdot d r \\
& \equiv \sum_{j=1}^{d-1} \sum_{k=1}^{d} v_{i}^{(j)} e_{i j}^{(k)} d C^{(k)}+r\left\langle v_{i}, d v_{i}\right\rangle+\left\langle v_{i}, v_{i}\right\rangle d r \\
& \equiv \sum_{k=1}^{d}\left(\sum_{j=1}^{d-1} v_{i}^{(j)} e_{i j}^{(k)}\right) d C^{(k)}+\left(\frac{r}{2}\right) \cdot d\left\langle v_{i}, v_{i}\right\rangle+d r
\end{aligned}
$$




$$
\begin{aligned}
& \equiv r^{-1} \sum_{k=1}^{d} \bar{P}_{i}^{(k)} d C^{(k)}+0+d r \\
& \equiv r^{-1}\left\langle\bar{P}_{i}, d C\right\rangle+d r .
\end{aligned}
$$

It follows that

$$
\begin{aligned}
\bigwedge_{i=0}^{d} \Sigma_{i} & \equiv \bigwedge_{i=0}^{d}\left(r^{-1}\left\langle\bar{P}_{i}, d C\right\rangle+d r\right) \\
& =r^{-d} \cdot\left|\begin{array}{cccc}
\bar{P}_{0}^{(1)} & \cdots & \bar{P}_{0}^{(d)} & 1 \\
\vdots & \ddots & \vdots & \vdots \\
\bar{P}_{d}^{(1)} & \cdots & \bar{P}_{d}^{(d)} & 1
\end{array}\right| d C^{(1)} \wedge \cdots \wedge d C^{(d)} \wedge d r \\
& =r^{-d}\left(d ! \cdot \lambda_{d}(\Delta)\right) d C^{(1)} \wedge \cdots \wedge d C^{(d)} \wedge d r
\end{aligned}
$$

where $\Delta$ represents the $d$-simplex with vertices $P_{0}, \ldots, P_{d}$.

A simple consequence of anticommutativity is that $d x \wedge d y=(\alpha d x+\beta d y) \wedge(d y / \alpha)$; so,

$$
\begin{aligned}
\bigwedge_{i=0}^{d} d \mu\left(L_{i}\right)= & \bigwedge_{i=0}^{d}\left(\bigwedge_{j=1}^{d-1} d Q_{i}^{(j)} \wedge d \sigma_{d-1}\left(u_{i}\right)\right) \\
= & \bigwedge_{i=0}^{d}\left(\Sigma_{i} \wedge \frac{1}{v_{i}^{(1)}} \bigwedge_{j=2}^{d-1} d Q_{i}^{(j)} \wedge d \sigma_{d-1}\left(u_{i}\right)\right) \\
= & \left(\bigwedge_{i=0}^{d} \Sigma_{i}\right) \wedge \bigwedge_{i=0}^{d}\left(\frac{1}{v_{i}^{(1)}} \bigwedge_{j=2}^{d-1} d Q_{i}^{(j)} \wedge d \sigma_{d-1}\left(u_{i}\right)\right) \\
= & d ! r^{-d} \lambda_{d}(\Delta) d C^{(1)} \wedge \cdots \wedge C^{(d)} \wedge d r \\
& \wedge \bigwedge_{i=0}^{d}\left(\frac{1}{v_{i}^{(1)}} \bigwedge_{j=2}^{d-1} r d v_{i}^{(j)} \wedge d \sigma_{d-1}\left(u_{i}\right)\right) \\
= & d ! r^{(d-2)(d+1)-d} \lambda_{d}(\Delta) d C^{(1)} \wedge \cdots \wedge d C^{(d)} \wedge d r \\
& \wedge \bigwedge_{i=0}^{d}\left(d \sigma_{d-2}\left(v_{i}\right) \wedge d \sigma_{d-1}\left(u_{i}\right)\right)
\end{aligned}
$$

Our last step is to express $C$ in terms of spherical coordinates $q=|C|$ and $w=C / q$ :

$$
\begin{gathered}
d \mu\left(L_{0}\right) \cdots d \mu\left(L_{d}\right)=d ! r^{(d-2)(d+1)-d} \lambda_{d}(\Delta) q^{d-1} d q d \sigma_{d-1}(w) d r \\
\wedge \bigwedge_{i=0}^{d}\left(d \sigma_{d-2}\left(v_{i}\right) d \sigma_{d-1}\left(u_{i}\right)\right)
\end{gathered}
$$




\section{Expectations}

Let $\mathrm{E} V_{n}$ represent the expected number of Voronoi vertices of $\mathcal{L}_{n}$. Since the lines are i.i.d.,

$$
\begin{aligned}
E V_{n} & =\left(\begin{array}{c}
n \\
d+1
\end{array}\right) \frac{\int\left(1-\varphi\left(L_{0}, \cdots, L_{d}\right)\right)^{n-d-1} d \mu\left(L_{0}\right) \cdots d \mu\left(L_{d}\right)}{\int d \mu\left(L_{0}\right) \cdots d \mu\left(L_{d}\right)} \\
& \sim \frac{n^{d+1}}{(d+1) !}\left(S_{d-1} S_{d-2}\right)^{-(d+1)} \int(1-\varphi)^{n-d-1} d \mu\left(L_{0}\right) \cdots d \mu\left(L_{d}\right),
\end{aligned}
$$

where $\varphi=\varphi\left(L_{0}, \ldots, L_{d}\right)$ represents the probability that a random line intersecting $B_{d}$ also intersects the random $d$-ball defined by $L_{0}, \ldots, L_{d}$, and integration is over all $(d+1)$-tuples of lines intersecting the unit $d$-ball.

Adopting the parametrization of the $d+1$ lines described in Section 3, we obtain

$$
\begin{aligned}
E V_{n} \sim & \frac{n^{d+1}}{(d+1) S_{d-1}^{d+1} S_{d-2}^{d+1}} \int(1-\varphi)^{n-d-1} \lambda_{d}(\Delta) q^{d-1} r^{(d-2)(d+1)-d} \\
& \times d r d q d \sigma_{d-1}(w) \bigwedge_{i=0}^{d}\left(d \sigma_{d-1}\left(u_{i}\right) d \sigma_{d-2}\left(v_{i}\right)\right)
\end{aligned}
$$

Nothing else depends on $w$, and we may integrate $\int d \sigma_{d-1}(w)=S_{d-1}$. The probability $\varphi\left(L_{0}, \ldots, L_{d}\right)$ depends only on $q$ and $r$, as do the integrals $\int d \sigma_{d-1}\left(u_{i}\right) d \sigma_{d-2}\left(v_{i}\right)$ for $0 \leq i \leq d$. In fact, for fixed $r$ and $q$, the quantity

$$
\frac{\int \lambda_{d}(\Delta) d \sigma_{d-1}\left(u_{0}\right) d \sigma_{d-2}\left(v_{0}\right) \cdots d \sigma_{d-1}\left(u_{d}\right) d \sigma_{d-2}\left(v_{d}\right)}{\int d \sigma_{d-1}\left(u_{0}\right) d \sigma_{d-2}\left(v_{0}\right) \cdots d \sigma_{d-1}\left(u_{d}\right) d \sigma_{d-2}\left(v_{d}\right)}=: \operatorname{E} \Delta(q, r)
$$

is the expected volume of the simplex formed by the points of tangency of $d+1$ random lines on a sphere of radius $r$ centered at distance $q$ from the origin. Since the $d+1$ lines are i.i.d., the denominator is equal to

$$
\left(\int d \sigma_{d-1}\left(u_{0}\right) d \sigma_{d-2}\left(v_{0}\right)\right)^{d+1}=: T(q, r)^{d+1}
$$

Finally, we estimate $(1-\varphi(q, r))^{n-d-1}$ by $\exp (-n \varphi(q, r))$, and we can write

$$
\begin{aligned}
\mathrm{E} V_{n} \sim & \frac{n^{d+1}}{(d+1) S_{d-1}^{d+1} S_{d-2}^{d+1}} \int_{0}^{\infty} \int_{0}^{\infty} \exp (-n \varphi(q, r)) \mathrm{E} \Delta(q, r) T(q, r)^{d+1} \\
& \times q^{d-1} r^{(d-2)(d+1)-d} d r d q .
\end{aligned}
$$

It is now only necessary to estimate $\varphi(q, r), \mathrm{E} \Delta(q, r)$, and $T(q, r)$ for the random ball defined by the $d+1$ random lines. We partition the range of integration into ten regions and apply different estimates in each. The regions differ in whether or not the center of the random ball lies inside or outside the unit ball, whether or not the random ball is smaller or larger than the unit ball, and how the intersection of the random ball and the unit ball is shaped. We write $\mathrm{E} V_{n}^{(i)}$ for the contribution of the $i$ th region, and now consider each: 
Region 1: $q \leq 1$ and $0 \leq r \leq 1-q$. In this region the entire random ball lies inside the unit ball, and every line tangent to or intersecting the random ball also intersects the unit ball. So $T(q, r)=S_{d-1} S_{d-2}, \varphi(q, r)=r^{d-1}$, and, since the points of tangency of the $d+1$ random lines are uniformly distributed on the random sphere, $\mathrm{E} \Delta(q, r)=\tau_{d} r^{d}$ for a constant $\tau_{d}$ computed by Miles [4]. We have

$$
\begin{aligned}
E V_{n}^{(1)} & \sim \frac{n^{d+1}}{d+1} \tau_{d} \int_{0}^{1} \int_{0}^{1-r} \exp \left(-n r^{d-1}\right) q^{d-1} r^{(d-2)(d+1)} d q d r \\
& \sim \frac{n^{d+1}}{d+1} \tau_{d} d^{-1} \int_{0}^{1} \exp \left(-n r^{d-1}\right)(1-r)^{d} r^{(d-2)(d+1)} d r \\
& \sim \frac{n^{d+1}}{d+1} \tau_{d} d^{-1} \int_{0}^{n} \exp (-t)\left(1-\left(\frac{t}{n}\right)^{1 /(d-1)}\right)^{d}\left(\frac{t}{n}\right)^{d(d-2) /(d-1)} \frac{d t}{(d-1) n} \\
& \sim n^{d /(d-1)} \frac{\Gamma\left(\left(d^{2}+1\right) / 2\right) \Gamma(d / 2)^{d+1}}{\sqrt{\pi} d(d-1)(d+1) ! \Gamma\left(d^{2} / 2\right) \Gamma((d+1) / 2)^{d}} \Gamma\left(d-\frac{1}{d-1}\right)
\end{aligned}
$$

Region 2: $q \leq 1$ and $1-q \leq r \leq 1$. In this region the intersection of the random ball and the unit ball contains a ball of radius $r / 2$, so $\varphi(q, r) \geq(r / 2)^{d-1}$. With the crude bounds $T(q, r)=O(1)$ and $\mathrm{E} \Delta(q, r)=O\left(r^{d}\right)$, we obtain

$$
\begin{aligned}
E V_{n}^{(2)} & =O\left(n^{d+1}\right) \int_{0}^{1} \int_{1-r}^{1} \exp \left(-n\left(\frac{r}{2}\right)^{d-1}\right) q^{d-1} r^{(d-2)(d+1)} d q d r \\
& =O\left(n^{d+1}\right) \int_{0}^{1} \exp \left(-n r^{d-1}\right) r^{1+(d-2)(d+1)} d r \\
& =O\left(n^{d+1}\right) \int_{0}^{n} \exp (-t)\left(\frac{t}{n}\right)^{d-1} \cdot \frac{d t}{n} \\
& =O(n) .
\end{aligned}
$$

Region 3: $q \leq 1$ and $1 \leq r \leq 2$. In this region the intersection of the random ball and the unit ball contains a ball of radius $\frac{1}{2}$, and thus $\varphi(q, r) \geq\left(\frac{1}{2}\right)^{d-1}$. Using the crude bounds $T(q, r)=O(1)$ and $\mathrm{E}_{\Delta}(q, r)=O\left(r^{d}\right)$, it is easy to show that

$$
E V_{n}^{(3)}=O\left(n^{d+1} \exp \left(-n 2^{1-d}\right)\right)=o(1) .
$$

Region 4: $q \leq 1$ and $r \geq 2$. In this region the unit ball is completely contained by the random ball. Since no lines tangent to the random ball pass through the unit ball, $T(q, r)=0$, and $\mathrm{E} V_{n}^{(4)}=0$.

Region 5: $q \geq 1, r \leq 1$, and $0 \leq r \leq q-1$. To estimate $\varphi(q, r)$ in this region, we consider the projections of the random and unit balls onto some fixed hyperplane. If the projected center of the random ball falls inside the projection of the unit ball, then the intersection of the projections contains a $(d-1)$-ball of radius $r / 2$. This happens precisely when the angle $\theta$ between the hyperplane normal and the line joining the centers of the two balls satisfies $\sin \theta \leq 1 / q$. Thus,

$$
\varphi(q, r) \geq \int_{0}^{\arcsin (1 / q)}\left(\frac{r}{2}\right)^{d-1} \sin ^{d-2} \theta d \theta
$$




$$
\begin{aligned}
& =\Omega\left(r^{d-1}\right) \int_{0}^{\arcsin (1 / q)} \theta^{d-2} d \theta \\
& =\Omega\left(r^{d-1}\left(\arcsin \left(\frac{1}{q}\right)\right)^{d-1}\right) \\
& =\Omega\left(\left(\frac{r}{q}\right)^{d-1}\right)
\end{aligned}
$$

To estimate $T(q, r)$, we consider the tangent lines passing through a fixed point $P$ on the random ball, and compute the fraction that also intersect the unit ball. If $C$ is the center of the random ball, the intersection is empty unless $\theta_{1} \leq \angle P C O \leq \theta_{2}$, where $\cos \theta_{1}=(r+1) / q$ and $\cos \theta_{2}=(r-1) / q$; these limits are determined by hyperplanes tangent to both balls. The intersection itself is a $(d-1)$-ball whose center is at distance $q \sin \theta$ from $P$. The angle subtended at $P$ by the intersection is at most $2 \arcsin (1 /(q \sin \theta))$, since the radius of the $(d-1)$-ball is at most 1 . Therefore,

$$
\begin{aligned}
T(q, r) & =O(1) \int_{\theta_{1}}^{\theta_{2}}\left(\int_{0}^{\arcsin (1 /(q \sin \theta))} \sin ^{d-3} \psi d \psi\right) \sin ^{d-2} \theta d \theta \\
& =O(1) \int_{\theta_{1}}^{\theta_{2}}(q \sin \theta)^{2-d} \sin ^{d-2} \theta d \theta \\
& =O\left(q^{2-d}\right)\left(\theta_{2}-\theta_{1}\right) \\
& =O\left(q^{2-d}\right)\left(\left(\frac{\pi}{2}-\theta_{1}\right)+\left(\theta_{2}-\frac{\pi}{2}\right)\right) \\
& =O\left(q^{2-d}\right)\left(\arcsin \left(\frac{1+r}{q}\right)+\arcsin \left(\frac{1-r}{q}\right)\right) \\
& =O\left(q^{1-d}\right) .
\end{aligned}
$$

Similarly,

$$
\mathrm{E} \Delta(q, r) \leq\left(r \cos \theta_{1}-r \cos \theta_{2}\right) r^{d-1}=O\left(\frac{r^{d}}{q}\right) .
$$

So Region 5 contributes at most

$$
\begin{aligned}
\mathrm{E} V_{n}^{(5)} & =O\left(n^{d+1}\right) \int_{1}^{\infty} \int_{0}^{1} \exp \left(-n\left(\frac{r}{q}\right)^{d-1}\right) \frac{r^{d}}{q} q^{(1-d)(d+1)} q^{d-1} r^{(d-2)(d+1)-d} d r d q \\
& =O\left(n^{d+1}\right) \int_{1}^{\infty} \int_{0}^{\infty} \exp (-t)\left(\frac{t}{n}\right)^{d(d-2) /(d-1)} q^{-2} \frac{d t}{n} d q \\
& =O\left(n^{d /(d-1)}\right) .
\end{aligned}
$$

Region 6: $q \geq 1, r \leq 1$, and $q-1 \leq r \leq q$. In this region we must also have $q \leq 2$, i.e., $q=\Theta(1)$. Also, $\varphi(q, r) \geq \varphi(2, r)$, and, arguing as for Region $5, \varphi(2, r)=$ $\Omega\left((r / q)^{d-1}\right)=\Omega\left(r^{d-1}\right)$. Since $T(q, r)=O(1)$ and $\mathrm{E} \Delta(q, r)=O\left(r^{d}\right)$,

$$
E V_{n}^{(6)}=O\left(n^{d+1}\right) \int_{0}^{1} \int_{1}^{r+1} \exp \left(-n r^{d-1}\right) q^{d-1} r^{(d-2)(d+1)} d q d r=O(n) .
$$


Region 7: $q \geq 1, r \geq 1$, and $q \leq r \leq q+1$. In this region the intersection of the random ball and the unit ball contains a ball of radius $\frac{1}{2}, \operatorname{so} \varphi(q, r) \geq\left(\frac{1}{2}\right)^{d-1}=\Omega(1)$ and $E V_{n}^{(7)}=o(1)$.

Region 8: $q \geq 1, r \geq 1$, and $q-1 \leq r \leq q$. In this region $\varphi(q, r) \geq \varphi(2,1)=\Omega(1)$ and $E V_{n}^{(8)}=o(1)$.

Region 9: $q \geq 1$ and $1 \leq r \leq q-1$. Estimation of $\varphi(q, r)$ is similar to Region 5, with the roles of the two balls reversed: If the projected center of the unit ball falls inside the projection of the random ball, then the intersection of the projections contains a $(d-1)$ ball of radius $\frac{1}{2}$. This happens precisely when the angle $\theta$ between the hyperplane normal and the line joining the centers of the two balls satisfies $\sin \theta \leq r / q$. Thus,

$$
\varphi(q, r) \geq \int_{0}^{\arcsin (r / q)}\left(\frac{1}{2}\right)^{d-1} \sin ^{d-2} \theta d \theta=\Omega\left(\left(\frac{r}{q}\right)^{d-1}\right) .
$$

When $r / q>\frac{1}{3}$, we have $\varphi(q, r)=\Omega(1)$, so such cases contribute $o(1)$ to $\mathrm{E} V_{n}^{(9)}$. When $r / q \leq \frac{1}{3}$,

$$
T(q, r)=O\left(q^{2-d}\left(\theta_{2}-\theta_{1}\right)\right)
$$

as in Region 5, but with $\theta_{1}=\arccos ((r+1) / q)$ and $\theta_{2}=\arccos ((r-1) / q)$. The power-series expansion

$$
\arcsin (z)=\sum_{\text {odd } k \geq 1} C_{k} z^{k}, \quad \text { where } \quad C_{k}=\frac{(-1)}{k}^{\lfloor k / 2\rfloor}\left(\begin{array}{c}
-\frac{1}{2} \\
\lfloor k / 2\rfloor
\end{array}\right),
$$

converges for $|z|<1$. Letting $x=r / q$ and $y=1 / q$, we have $\cos \theta_{1}=x+y$, $\cos \theta_{2}=x-y$, and

$$
\begin{aligned}
\theta_{2}-\theta_{1} & =\arccos (x-y)-\arccos (x+y) \\
& =\arcsin (x+y)-\arcsin (x-y) \\
& =\sum_{\text {odd } k \geq 1} C_{k}\left((x+y)^{k}-(x-y)^{k}\right) \\
& \leq \sum_{\text {odd } k \geq 1} C_{k} \cdot\left(2^{k} x^{k-1} y^{1}\right) \\
& =\left(\frac{y}{x}\right) \sum_{\text {odd } k \geq 1} C_{k}(2 x)^{k} \\
& =\left(\frac{y}{x}\right) \arcsin (2 x) \\
& \leq \pi y=O\left(q^{-1}\right),
\end{aligned}
$$

and $T(q, r)=O\left(q^{1-d}\right)$. Finally, we have

$$
\mathrm{E} \Delta(q, r) \leq\left(r \cos \theta_{2}-r \cos \theta_{1}\right)\left(r \sin \theta_{2}\right)^{d-1}=O\left(\frac{r^{d}}{q}\right) .
$$

All three estimates are similar to Region 5's, and we can easily show that $E V_{n}^{(9)}=$ $O\left(n^{d /(d-1)}\right)$. 
Region 10: $q \geq 1$ and $r \geq q+1$. In this region $T(q, r)=0$ and therefore $\mathrm{E} V_{n}^{(10)}=0$. Summing over all ten regions, we see that $\mathrm{E} V_{n}=\Theta\left(n^{d /(d-1)}\right)$.

\section{Higher-Dimensional Flats}

Points and lines are zero- and one-dimensional flats, i.e., affine subspaces. This section briefly explores the combinatorial complexity of Voronoi diagrams of sets of random higher-dimensional flats. We outline the proof of the following theorem:

Theorem 2. For any fixed $d \geq 3$ and $0 \leq k \leq d-1$, the expected combinatorial complexity of the Euclidean Voronoi diagram of $n$ random $k$-flats drawn independently from the uniform distribution on $k$-flats intersecting the unit ball in $\mathbb{R}^{d}$ is $\Theta\left(n^{d /(d-k)}\right)$ as $n \rightarrow \infty$.

To choose a random $k$-flat, we first choose its orientation uniformly. Its orientation can be defined by $k$ pairwise orthogonal unit vectors $u_{1}, u_{2}, \ldots, u_{k}$; we choose $u_{1}$ according to the uniform measure $\sigma_{d-1}, u_{2}$ in the hyperplane normal to $u_{1}$ according to measure $\sigma_{d-2}, u_{3}$ in the $(d-2)$-flat normal to both $u_{1}$ and $u_{2}$ according to $\sigma_{d-3}$, etc. A $k$-flat $L$ (hereafter "flat") with a fixed orientation is uniquely determined by its intersection with the unique $(d-k)$-flat (hereafter "co-flat") orthogonal to $L$ and passing through the origin; for translation-invariance, we choose the intersection point uniformly on the co-flat according to the Lebesgue measure $\lambda_{d-k}$. Thus, the uniform measure on $k$-flats in $\mathbb{R}^{d}$ satisfies [6, p. 204]

$$
d \mu_{d, k}(L)=d \lambda_{d-k}(P) \wedge d \sigma_{d-1}\left(u_{1}\right) \wedge \cdots \wedge d \sigma_{d-k}\left(u_{k}\right) .
$$

For brevity, we write $d \sigma_{d, k}\left(u_{1}, \ldots, u_{k}\right)$ for $d \sigma_{d-1}\left(u_{1}\right) \wedge \cdots \wedge d \sigma_{d-k}\left(u_{k}\right)$.

As before, we must develop a density transformation. Each vertex of the Voronoi diagram is (with probability 1 ) equidistant from $d+1$ flats. If $C$ is the vertex and $r$ is the distance to each line, then the $d+1$ flats are tangent to a $(d-1)$-sphere of radius $r$ centered at $C$.

Let $U_{i}=\left(u_{i 1}, \ldots, u_{i k}\right)$ consist of $k$ pairwise orthogonal unit vectors lying on flat $L_{i}$, and let $H_{i}=\left\{x \mid\left\langle u_{i 1}, x\right\rangle=\cdots=\left\langle u_{i k}, x\right\rangle=0\right\}$ be $L_{i}$ 's co-flat through the origin. Let $\left(e_{i 1}, e_{i 2}, \ldots, e_{i, d-k}\right)$ be a moving frame for $H_{i}$. Let $P_{i} \in \mathbb{R}^{d}$ be the vector of coordinates of $L_{i} \cap H_{i}$ with respect to the standard basis, and let $Q_{i} \in \mathbb{R}^{d-k}$ be its coordinates with respect to the moving frame, so that $Q_{i}^{(j)}=\left\langle P_{i}, e_{i j}\right\rangle$. Let $\bar{H}_{i}=\left\{x \mid\left\langle u_{i j}, x\right\rangle=\right.$ $\left\langle u_{i j}, C\right\rangle$ for $\left.1 \leq j \leq k\right\}$ be the co-flat perpendicular to flat $L_{i}$ passing through $C$. Let $\bar{P}_{i}$ be the vector of coordinates of $\left(L_{i} \cap \bar{H}_{i}\right)-C$ with respect to the standard basis for $\mathbb{R}^{d}$, and let $\bar{Q}_{i}$ be its coordinates with respect to the moving frame. Finally, let $v_{i}=\bar{Q}_{i} /\left|\bar{Q}_{i}\right|$ be a unit vector in $\mathbb{R}^{d-k}$.

To express $\bigwedge_{i=0}^{d} d \mu_{d, k}\left(L_{i}\right)$ in terms of the $d u_{i k}^{(j)}, d C^{(j)}, d r$, and $d v_{i}^{(j)}$, we proceed as in Section 3. Equations (3.1) and (3.2) still hold for $1 \leq j \leq d-k$, so we define

$$
\Sigma_{i}=\sum_{j=1}^{d-1} v_{i}^{(j)} d Q_{i}^{(j)} \equiv r^{-1}\left\langle\bar{P}_{i}, d C\right\rangle+d r
$$


and find that (3.3) is valid. We eventually obtain

$$
\begin{aligned}
\bigwedge_{i=0}^{d} d \mu_{d, k}\left(L_{i}\right)= & \bigwedge_{i=0}^{d}\left(\bigwedge_{j=1}^{d-k} d Q_{i}^{(j)} \wedge d \sigma_{d, k}\left(U_{i}\right)\right) \\
= & \left(\bigwedge_{i=0}^{d} \Sigma_{i}\right) \wedge \bigwedge_{i=0}^{d}\left(\frac{1}{v_{i}^{(1)}} \bigwedge_{j=2}^{d-k} r d v_{i}^{(j)} \wedge d \sigma_{d, k}\left(U_{i}\right)\right) \\
= & d ! r^{(d-k-1)(d+1)-d} \lambda_{d}(\Delta) q^{d-1} d q \wedge d \sigma_{d-1}(w) \wedge d r \\
& \wedge \bigwedge_{i=0}^{d}\left(d \sigma_{d-k-1}\left(v_{i}\right) \wedge d \sigma_{d, k}\left(U_{i}\right)\right) .
\end{aligned}
$$

Arguments like Section 4's show that

$$
\begin{aligned}
\mathrm{E} V_{n}= & \Theta\left(n^{d+1}\right) \int_{0}^{\infty} \int_{0}^{\infty} \exp (-n \varphi(q, r)) \mathrm{E} \Delta(q, r) T(q, r)^{d+1} q^{d-1} \\
& \times r^{(d-k-1)(d+1)-d} d r d q
\end{aligned}
$$

where $\varphi(q, r)$ is the probability that a random $k$-flat intersecting the unit ball also intersects the random ball, $E \Delta(q, r)$ is the expected volume of the simplex formed by the $d+1$ points of tangency on the random ball, and

$$
T(q, r):=\int d \sigma_{d, k}\left(U_{0}\right) d \sigma_{d-k-1}\left(v_{0}\right)
$$

with integration over all flats tangent to the random ball that also intersect the unit ball.

We consider the ten regions of integration as before. In Region 1, where the random ball is entirely contained in the unit ball, $\varphi(q, r)=r^{d-k}, E \Delta(q, r)=\tau_{d} r^{d}, T(q, r)=$ $S_{d-1} S_{d-2} \cdots S_{d-k-1}$, and $E V_{n}^{(1)}=\Theta\left(n^{d /(d-k)}\right)$.

Estimating $\varphi(q, r)$ in Region 5 is a computation of more typical difficulty. To compute this probability exactly, we would integrate over all possible co-flats through the origin; the integrand would be the $(d-k)$-volume of the intersection of the projections of the unit and random balls onto the co-flat. However, a lower bound can be obtained by computing the measure of co-flats for which the center of the random ball is projected into the unit ball; in each such case, the intersection of the projected balls contains a $(d-k)$-ball of radius $r / 2$. To measure such co-flats, it is easier to fix the co-flat to be $x^{(d-1)}=x^{(d-2)}=\cdots=x^{(d-k+1)}=0$, then to allow the center of the random ball to range over a $(d-1)$-sphere of radius $q$ centered at the origin; the quantity of interest is the probability that the center satisfies $\sum_{i=1}^{d-k}\left(x^{(i)}\right)^{2} \leq 1$. Equivalently, we may choose a random unit vector $u$ on the $(d-1)$-sphere and find the probability that $\sum_{i=1}^{d-k}\left(u^{(i)}\right)^{2} \leq q^{-2}$. With the usual spherical coordinates

$$
\begin{aligned}
u^{(1)} & =s_{d-1} s_{d-2} \cdots s_{3} s_{2} s_{1} c_{0}, \\
u^{(2)} & =s_{d-1} s_{d-2} \cdots s_{3} s_{2} c_{1}, \\
u^{(3)} & =s_{d-1} s_{d-2} \cdots s_{3} c_{2}, \\
& \vdots \\
u^{(d)} & =c_{d-1}, \\
d \sigma_{d-1}(u) & =s_{d-1}^{d-2} \cdots s_{1}^{0} d \psi_{d-1} \cdots d \psi_{1},
\end{aligned}
$$


with $c_{0}=1, c_{i}=\cos \psi_{i}$, and $s_{i}=\sin \psi_{i}$, we have

$$
\sum_{i=1}^{d-k}\left(u^{(i)}\right)^{2}=\left(s_{d-1} s_{d-2} \cdots s_{d-k}\right)^{2} \leq s_{d-k}^{2} .
$$

So

$$
\begin{aligned}
\varphi(q, r) & \geq \frac{(r / 2)^{d-k}}{S_{d-1}}\left(\prod_{\substack{1 \leq i \leq d-1 \\
i \neq d-k}} \int_{0}^{\pi / 2} s_{i}^{i-1} d \psi_{i}\right)\left(\int_{0}^{\arcsin (1 / q)} s_{d-k}^{d-k-1} d \psi_{d-k}\right) \\
& =\Omega\left(\left(\frac{r}{q}\right)^{d-k}\right) .
\end{aligned}
$$

It is straightforward but tedious to show that $\varphi(q, r)=\Theta\left((r / q)^{d-k}\right)$ in Region 5 and to complete the analysis of this and the other eight regions along the lines of Section 4.

\section{Final Remarks}

While we have determined the asymptotic order of the expected number of Voronoi vertices, we have also raised some other questions:

Constant Factors. It would be satisfying to know with precision the leading constant factor (for fixed $d$ and $k$ ), or at least to know its asymptotic behavior as $k$ and/or $d$ grow. In fact, the leading constant $i$ given precisely for Region 1 . With some additional effort, it is possible to show that the upper bounds given for Regions 5 and 9 are in fact tight up to asymptotic order. Therefore, rather exact estimates of $\varphi, T$, and $\mathrm{E} \Delta$ are required in these regions to derive the leading constant of $E V_{n}$. Such estimates are unwieldy, even for the relatively simple case of lines.

Furthest-Site Voronoi Diagrams and Extreme Flats. A more interesting question relates to average complexity of the furthest-site Voronoi diagrams induced by the function

$$
V_{F}(P)=\left\{L \in \mathcal{L}_{n} \mid \delta(P, L) \geq \delta\left(P, L^{\prime}\right), \forall L^{\prime} \in \mathcal{L}_{n}\right\} .
$$

When point sites are considered, only points on the convex hull generate cells of the furthest-site diagram. For points uniform in the $d$-ball, it is known that there are $\Theta\left(n^{(d-1) /(d+1)}\right)$ convex-hull sites on average [5]. We conjecture that the number of furthest-site Voronoi vertices has the same asymptotic order.

When the sites are lines or higher-dimensional flats, the convex hull concept is not applicable. Still, we conjecture that the number of flats generating cells of the furthestsite Voronoi diagram is $o(n)$, and that the number of Voronoi vertices has the same asymptotic order. We suggest that the flats that generate Voronoi cells be regarded as the "extreme flats" of the set.

The methods of this paper are applicable to the furthest-site problem by replacing the expression $1-\varphi(q, r)$ with $\varphi(q, r)$ in (4.1). The difficulty comes in making suitable estimates of $\varphi(q, r)$, since the leading constant factors are crucial even for determining asymptotic order. 
Dynamic Voronoi Diagrams. The Voronoi diagram of lines in $\mathbb{R}^{d+1}$ is related to the Voronoi diagram of points moving through $\mathbb{R}^{d}$ with constant velocity [1]. As time progresses, the geometric structure of the diagram changes continuously, but its combinatorial structure changes only at $O\left(n^{d+2}\right)$ instants when $d+2$ points lie on a common $(d-1)$-sphere. The overall complexity of the dynamic diagram may be regarded as the number of cells at time $t=0$ plus the number of combinatorial changes as the diagram evolves. It is not too difficult to derive an appropriate density transformation for $d+2$ moving points on a $(d-1)$-sphere, but the rest of the analysis appears to be challenging.

\section{References}

1. G. Albers and T. Roos. Voronoi diagrams of moving points in higher dimensional spaces. Proc. 3rd Scandinavian Workshop on Algorithm Theory, pages 399-409, July 1992.

2. L. P. Chew, K. Kedem, M. Sharir, B. Tagansky, and E. Welzl. Voronoi diagrams of lines in 3-space under polyhedral convex distance functions. Proc. 6th Ann. ACM-SIAM Symp. on Discrete Algorithms, pages $197-$ 204, January 1995.

3. R. A. Dwyer. Higher-dimensional Voronoi diagrams in linear expected time. Discrete Comput. Geom., 6(4):343-367, 1991.

4. R. E. Miles. Isotropic random simplices. Adv. in Appl. Probab., 3:353-382, 1971.

5. H. Raynaud. Sur l'envelope convexe des nuages des points aléatoires dans $\mathbb{R}^{n}, 1$. J. Appl. Probab., 7:35-48, 1970.

6. L. A. Santal6. Integral Geometry and Geometric Probability. Encyclopedia of Mathematics and Its Applications, volume 1. Addison-Wesley, Reading, MA, 1976.

7. M. I. Shamos and D. Hoey. Closest-point problems. Proc. 16th Symp. on Foundations of Computer Science, pages 151-162, 1975.

Received March 15, 1995, and in revised form November 13, 1995. 